\title{
Responsabilidad profesional frente al aborto
}

\author{
Jorge Villarreal, M.D.*
}

RESUMEN: La mortalidad materna por aborto continúa siendo muy elevada en Colombia y el subregistro, que es del $50 \%$ para la mortalidad materna general, debe ser aún mayor en el caso del aborto.

Tal como se ha demostrado en los países donde el aborto ha sido despenalizado, las muertes ocasionadas por complicaciones secundarias al aborto son casi totalmente evitables.

En Colombia donde la despenalización del aborto es muy poco probable en el futuro previsible, la mortalidad materna por aborto continuará siendo muy elevada debido a la alta prevalencia del embarazo indeseado, el lento progreso en la tecnología anticonceptiva y las múltiples barreras que limitan la efectividad de los programas de planificación familiar.

Los obstetras ginecólogos deben contribuir a la solución de este grave problema no sólo denunciando su existencia sino con acciones concretas en su práctica profesional, en la docencia y estimulando cambios en las leyes existentes.

PALABRAS ClAVES: Aborto, embarazo no deseado, ética profesional, mortalidad materna.

SUMMARY: Maternal mortality due to abortion continues to be high in Colombia and at least one of every two maternal deaths are not registered in the official statistics but underreporting of abortion deaths should be even higher.

Abortion is one of the most frecuent causes of maternal mortality, but few recognize that those are clearly avoidable deaths as shown in countries were abortion has been legalized.

Due to social and cultural dynamics of Colombian society, slow progress in contraceptive techonology and the multiple barriers that limit efectiveness of family planning programs, elevated maternal morbidity and mortality resultant from undesired pregnancy and abortion will continue to be extremely frequent in this country where depenalization of abortion is not likely to occur in the near future.

Obstetricians and Gynecologists, should contribute to the solution of this problem, not just by denouncing its prevalence but through concrete actions in their medical practice, teaching programs and influence over existing laws.

KEY WORDS: Abortion, undesired pregnancy, professional ethics, maternal mortality

Estudios aún no publicados en diciembre de 1991 sobre mortalidad materna y perinatal, realizados por las Sociedades de Obstetricia y Ginecología del Valle y de Antioquia (1) me permiten dar una perspectiva epidemiológica al análisis de la responsabilidad del obstetra-ginecólogo frente al aborto y resumir en ocho puntos la esencia de mi argumentación.

\section{Elevada mortalidad materna por aborto}

La mortalidad materna continúa siendo muy elevada en Colombia y dentro de sus causas se destaca en segundo lugar EL ABORTO que en otros países latinoamericanos sigue ocupando el primer lugar.

Es así como en seis meses de 1990 en 15 hospitales de Colombia ocurrieron 21 muertes maternas por aborto y el período 1985-89 en una sola ciudad, Cali, ocurrieron 40, un promedio de 8 por año (2).

\section{Subregistro de la mortalidad}

Los estudios llevados a cabo en Medellín y en Cali bajo la dirección de los doctores A. Jubiz, L.O. Castaño y C. Quintero ponen de presente un subregistro de la mortalidad

* Director Ejecutivo ORIENTAME-Santafé de Bogotá-Colombia. materna del $50 \%$. Es muy probable que dado el estigma y la penalización, el subregistro sea mucho mayor en la mortalidad por aborto.

\section{Interrogantes y respuestas}

Es responsabilidad del epidemiólogo preguntarse ¿de qué se muere la gente? y también ¿por qué se muere la gente?

Con base en múltiples estudios repetidos desde hace varias décadas, publicados la gran mayoría en la revista de la especialidad, la respuesta a la primera pregunta aplicada a la mujer en edad fértil es que ellas se mueren de aborto y a la segunda, no expresada en las publicaciones antedichas pero evidente en el más superficial de los análisis es que las mujeres se mueren porque el aborto es ilegal y clandestino.

\section{4. ¿Cúal es la causa básica de la mortalidad materna por aborto?}

El embarazo indeseado de altísina frecuencia estimulado por cambios socioculturales, es el evento que desencadena el proceso que lleva a la mujer a la muerte.

\section{5. ¿Cuál es el factor determinante de la mortalidad materna por aborto?}

Usualmente se mencionan múltiples factores (hasta 80 ), pero se ignora el factor primario directamente relacionado 
con la causa básica y origen de todos los demás. En Colombia el factor determinante de la alta mortalidad por aborto es la legislación anacrónica que penaliza el aborto y lo hace siempre ilegal.

\section{Evitabilidad de la mortalidad materna por aborto.}

Mientras que el embarazo indeseado y el aborto inducido son difícilmente evitables como se deduce de la elevada persistencia del mismo en países que por muchos años han tenido avanzados programas de educación sexual (ejemplo: Escandinavia) y amplia disponibilidad de métodos anticonceptivos (USA), lo que sí es evitable es la mortalidad asociada al aborto, hecho extensamente documentado en todos los países donde el aborto está legalizado.

\section{7. ¿Qué estrategias podrían aplicarse para la dismi- nución de la mortalidad materna por aborto?}

Todas las posibles estrategias deberían partir de la aplicación del concepto de riesgo al afirmar que "toda mujer que tiene un embarazo indeseado o inoportuno está de hecho en alto riesgo de tener un aborto inducido; y donde éste es ilegal, la mujer tiene un altísimo riesgo de enfermarse y morir por la misma causa".

\section{8. ¿Cuál es el futuro?}

Dada la dinámica económica y social de Colombia y de América Latina es válido asegurar que el embarazo indeseado y el aborto continuarán en aumento por varias décadas y de persistir la legislación vigente, lo cual es también muy probable, la mortalidad materna por aborto continuará también ocupando los primeros lugares.

La palabra $\mathrm{ABORTO}$ incomoda y el tema muy rápidamente polariza la discusión entre quienes dicen estar a FAVOR o en CONTRA, declarando así de un golpe, una posición no siempre reflexiva sino más bien influenciada por principios médicos tradicionales, vivencias junto a la joven mujer agonizante o postulados religiosos expresados con carácter dogmático.

Realmente, nadie está a FAVOR del aborto. Ni la mujer que recurre a él como último recurso frente a un embarazo no deseado, ni la persona que lo ejecuta, médico o no, unos con ética y responsabilidad profesional, otros por dinero, pero ninguno por que de verdad esté a favor del aborto.

Quienes dicen estar en CONTRA del aborto lo hacen pensando en "tercerá persona" y casi nunca enfrentados, personalmente las mujeres, o los hombres como padres, a través de su relación de pareja, o desde cualquier posición oficial, profesional o académica, a la tragedia de un embarazo inoportuno o no deseado.

Gran parte de la controversia suscitada en la opinión pública por el aborto, se genera en el desconocimiento de sus verdaderas raíces, el embarazo indeseado, y en el análisis superficial centrado en frases estereotipadas como "el egoísmo de la mujer", el "afán de lucro de los médicos", "la elevada mortalidad y morbilidad materna" o "el derecho a la vida del feto".

Todos esos enfoques parciales y muchísimos más, pueden dar lugar a discusiones científicas, eruditas, cargadas de emotividad y sentimientos encontrados, pero pocas veces útiles a quien es el sujeto de nuestra profesión y especialidad médica: La mujer y su entorno como madre, pareja y miembro de una sociedad.

El estudio realizado por la Federación Colombiana de Obstetricia y Ginecología en 1990 como parte de una investigación regional de la FLASOG (2), es el más reciente de una larga serie de trabajos descriptivos sobre el aborto que han sido publicados en la Revista de la especialidad en los últimos 25 años. En todos ellos se ha puesto de presente la responsabilidad prioritaria del aborto en la mortalidad materna y se han formulado conclusiones y recomendaciones que con sinceridad han buscado atacar las raíces del problema; veamos algunas de ellas:

\section{"Soluciones:}

Queremos insistir nuevamente en la necesidad de afrontar sin demora el problema del aborto provocado. Lograr una nueva estructura social que modifique conceptos básicos inculcando saber y moral, parece ser el punto clave...

En tanto que una realización así se desarrolla es necesario luchar contra las abortadoras...".

Rev. Col. Obs/Gyn

18 (2): 381-404, 1967

"Se recomienda estudiar la posibilidad de adoptar a nivel nacional una clasificación".

Rev. Col. Obs/Gyn

21 (2): 147-170, 1970

"Recomendaciones: A) Educación: Encaminada a educación sexual en las escuelas secundarias. B) Planificación familiar: Ampliando el área de cobertura a todos los niveles."

Rev. Col. Obs/Gyn 22 (4): 2333-246, 1971

"El aborto constituye en la actualidad un problema médico social de gran magnitud."

Rev. Col. Obs/Gyn 25 (2): 127-154, 1974

"La mayoría de estas muertes son prevenibles con una adecuada atención prenatal, un mejor manejo de las complicaciones del parto y con campañas educativas"

Rev. Col. Obs/Gyn 27 (3): 129-139, 1976

"Para tratar de sacar algún provecho práctico del estudio de la mortalidad materna se hace necesario establecer un juicio de responsabilidades".

Rev. Col. Obs/Gyn 28 (6): 217-229, 1977 
"Para reducir considerablemente la mortalidad por aborto, se deben difundir programas de planificación familiar y educar a la población. En los países donde es permitido el aborto, se piensa que su legalización ha sido un factor determinante en la reducción de la mortalidad materna".

$$
\begin{array}{r}
\text { Rev. Acta Médica del Valle } \\
10(3-4), 1979
\end{array}
$$

"El aborto séptico continúa siendo un problema grave en los servicios de obstetricia..."

"Finalmente afirmamos no poder ofrecer solución definitiva al problema por cuanto el aspecto médico no es factor determinante y corresponde al Estado dar solución a los factores socio-económicos y culturales..."

Rev. Col. Obs/Gyn

32 (2): 104-134, 1981

"Al igual que en toda la literatura que sobre el tema se ha escrito en Colombia en los últimos diez años, la primera causa de mortalidad materna sigue siendo la sepsis (44.8\%) y de ésta, la sepsis por aborto..."

Rev. Col. Obs/Gyn 33 (5): 325-336, 1982

"Mejorar el conocimiento y la accesibilidad de los métodos anticonceptivos..."

"Aumentar, promover y en algunos casos incluir la educación sexual en todas las instituciones docentes...."

Rev. Col. Obs/Gyn

42 (4): $287-97,1991$

\section{¿Cuál ha sido el efecto real de todos los esfuerzos consignados en dichos trabajos?}

A pesar de múltiples estudios, de la prescripción de esquemas de tratamiento del aborto séptico cada vez más avanzados, de la amplia disponibilidad de métodos anticonceptivos a bajo costo y la consecuente elevada prevalencia en el uso de éstos, el aborto continúa siendo muy frecuente y las mujeres colombianas continúan muriendo a consecuencia del mismo; en menor número que hace diez o veinte años, es cierto, pero todavía sufriendo la angustia, la explotación y la enfermedad resultante de un hecho que precisamente por ser clandestino, está rodeado de factores negativos e indeseables pero esencialmente prevenibles.

Además de su posible interés médico, epidemiológico o científico, el tema del aborto debe ser materia de reflexión personal por parte del especialista en obstetricia y ginecología. No sólo por las posibles graves consecuencias y por su elevada frecuencia, sino también como serio interrogante ante su propia conciencia.

¿Cuál es la posición, la actitud de cada uno de nosotros frente al aborto? ¿Qué estamos haciendo para disminuir su morbilidad?

¿Tenemos interés en algo que atañe tan de cerca a la mayoría de nuestras pacientes? ¿O preferimos aislarnos, ignorar el problema y nuestra responsabilidad profesional?

Hace doce años la Sociedad Colombiana de Ginecología y Obstetricia llevó a cabo en una muestra representativa de los médicos de Bogotá una encuesta anónima de actitudes de respuesta en relación con el aborto. ${ }^{(3)}$ Vale la pena hoy, doce años más tarde, recordar cuáles fueron las respuestas dadas a la pregunta que exploraba cuál sería la actitud personal de cada uno si la práctica del aborto fuera permitida por la ley:

Si bien $10 \%$ de nuestros colegas declararon que no practicarían el aborto bajo ninguna circunstancia, $60 \%$ dijeron que lo remitirían a otros médicos y cerca de $25 \%$ afirmaron que lo realizarían personalmente.

A partir del estudio realizado por FECOLSOG en quince hospitales de Colombia y de los hallazgos de un estudio patrocinado por la Organización Mundial de la Salud en ORIENTAME, centro médico privado de Bogotá, haré algunas consideraciones que tienen por objeto estimular la reflexión y fundamentar las afirmaciones que he formulado en las páginas precedentes.

El estudio sobre aborto hospitalario llevado a cabo por FECOLSOG nos revela una parte del problema:

\section{Mortalidad materna por aborto en Colombia (2)}

* Registros de 15 hospitales.

* 4263 abortos hospitalizados.

* 21 muertes maternas. (Estimadas 42 por año)

Pero de él surgen múltiples interrogantes:

* ¿Son éstas todas las muertes por aborto ocurridas en el país?

* ¿Cuáles son las consecuencias sociales de esta situación?

* ¿Es éste todo el problema médico secundario resultante del aborto ilegal y clandestino?

* Está usted interesado:

- ¿En conocer un poco más del problema?

- ¿En contribuir a su solución?

* ¿Se ha preguntado qué puede usted hacer?

Es indudable que la aparición de técnicas mejoradas de evaluación uterina en manos de profesionales, unidas a la formulación preventiva de antibióticos, debe estar incidiendo en la disminución de la mortalidad por aborto, pero surge la duda de si dicho efecto ha sido tan marcado como para ocasionar solamente 42 muertes maternas por aborto en todo el país cuando hace diez años un sólo hospital, el Instituto Materno Infantil de Bogotá, registraba 30 o más muertes por año.

Muchas mujeres que abortan dentro de las condiciones imperantes de clandestinidad, no mueren gracias a la disponibilidad de mejores servicios, pero ¿qué decir de 
la morbilidad evidente u oculta, secundaria a maniobras abortivas realizadas por personal no capacitado y las consecuencias sociales que ellas conllevan para la mujer y su familia?

El estudio de Morbilidad y Mortalidad Materna por Aborto en algunos hospitales realizado por la Federación Colombiana de Obstetricia y Ginecología, registra 4263 abortos y 21 defunciones maternas correspondientes a una tasa de 493 por cien mil abortos.

Tasa de Mortalidad Materna 21 muertes

Por aborto en Colombia $\quad-------\times 100.000=493$

4263 abortos

Este elevado número de muertes es evitable como lo demuestra una simple comparación con datos de países como los Estados Unidos de Norteamérica donde el aborto está legalizado y por lo mismo se puede suponer que todos los abortos quedan registrados en las estadísticas de salud. (4)

En dicho país la tasa de mortalidad materna es de 0.4 por cien mil abortos, y para establecer comparaciones significativas, debemos calcular cuál puede ser el número de estos ocurridos en el mismo período y en las mismas ciudades colombianas que no requirió hospitalización, por cada aborto que sí requirió ser hospitalizado.

Si estimamos que por cada aborto registrado en el estudio de FECOLSOG/FLASOG (4263), ocurrieron 50 abortos que no llegaron a los mismos hospitales, la Tasa de Mortalidad Materna por aborto sería 9.85 por cien mil, es decir veinticinco veces mayor que la de los Estados Unidos (0.4), pero si el número de los abortos que NO llegan a nuestros hospitales es solamente diez por cada uno de los registrados en el estudio, la Tasa de Mortalidad materna por aborto sube a 49 por cien mil, es decir 125 veces mayor que la de los Estados Unidos.

¿Qué ha pasado entre nosotros durante el último cuarto de siglo?

¿Ha disminuido el problema en alguna medida?

Como lo muestra la tabla Número 1, la mortalidad por aborto en relación con el número de éstos registrados en los hospitales continúa siendo muy alta, muchas veces superior inclusive a la de países vecinos y a veces mayor que hace 25 años.

TABLA No. 1

MORTALIDAD MATERNA

EN EL INSTITUTO MATERNO INFANTIL DE BOGOTA

\begin{tabular}{|ccccc|}
\hline \multicolumn{5}{c|}{25 años } \\
\hline Año & Abortos & $\begin{array}{l}\text { Muertes } \\
\text { Maternas }\end{array}$ & $\begin{array}{l}\text { Muertes } \\
\text { por Aborto }\end{array}$ & $\begin{array}{l}\text { TASA } \\
\times 100.0 C\end{array}$ \\
\hline $1966^{*}$ & 5555 & 77 & 25 & 450 \\
$1990^{* *}$ & 1042 & 21 & 8 & 768 \\
\hline
\end{tabular}

* López R. Rafael Rev. Col. Obs/Gyn. 19:269, 1968

** Datos estimados para 12 meses a partir del Estudio FLASOG (MarzoAgosto 1990).

El aborto continúa siendo un grave problema social, de salud individual y colectiva. ¿Estamos interesados en su solución? ¿Nos preguntamos cuál puede ser nuestro aporte?
A juzgar por los resultados del reciente estudio de aborto hospitalario llevado a cabo por FECOLSOG, la respuesta a ambos interrogantes no parece ser afirmativa cuando vemos que mientras $42 \%$ de las mujeres que ingresaron por aborto a los quince hospitales del estudio declararon NO desear ningún hijo más y a pesar de que 63 de cada cien no habían utilizado ningún método, solamente $37 \%$ recibieron alguna prescripción o servicio anticonceptivo en los mismos hospitales.

El problema generado por el embarazo indeseado y el aborto ilegal y clandestino tiene múltiples aspectos, muchos de ellos ignorados por los médicos que solamente reciben en la facultad de medicina información sobre lo que tiene que ver con la parte estrictamente médica del asunto. Me referiré a dos de ellas.

Una faceta pocas veces tenida en cuenta en relación con el aborto es el costo en términos económicos que para los hospitales y la salud pública del país representa el aborto clandestino.

A falta de estudios completos de costos hospitalarios, algunos datos parciales nos pueden dar somera idea del beneficio que para presupuestos municipales o estatales podría tener un cambio radical de política en el manejo del embarazo indeseado:

Quinientas veintiuna pacientes hospitalizadas en seis meses de 1991 en el Instituto Materno Infantil de Bogotá requirieron:

* 937 días de hospitalización.

Costo promedio de día $\$ 19.480$ (1990) . \$ 18.252 .760

* 1698 frascos de líquidos intravenosos.

$\mathrm{Col} \$ 300 \times \mathrm{fco}=\ldots \ldots . . . .$.

* 123 unidades (frascos de 500cc) de sangre.

$\$ 9.000$ por unidad de $500 \mathrm{cc}$. . . \$ 1.107 .000

* 260 horas de consulta de admisión.

* Sala de cirugía:

- 526 legrados.

- 7 cirugías adicionales.

* OTROS

* Estudio de Aborto Hospitalario. (2)

FECOLSOG/ FLASOG, 1990

Otro aspecto sin duda ignorado, soslayado y de todas maneras motivo de indiferencia para quien no quiere incomodarse pensando en el aborto ejercido en las condiciones de clandestinidad imperantes en Colombia, son aquellos costos, no necesariamente económicos, que afrontan tanto la mujer que aborta como su familia inmediata:

* Búsqueda angustiosa de servicio seguro y a costo accesible.

* Humillación y extorsión en servicio no humanizado.

* Temor al dolor, enfermedad y muerte.

* Costos generalmente por encima de su capacidad económica.

* Hospitalización forzada por técnicas inadecuadas.

* Abandono de hijos y hogar durante hospitalización evitable.

* Morbilidad y mortalidad de acuerdo con el tipo de servicio obtenido.

Costos todos ellos medidos en sufrimiento, humillación y a veces en la única forma en que parecería que los médicos 
sabemos medir el sufrimiento humano: En enfermedad física y muerte.

Ha sido lugar común de todos los estudios, análisis y publicaciones sobre aborto a partir de la aparición de la anticoncepción, el propugnar por programas de planificación familiar cada vez mejores y por casi treinta años los médicos venimos afirmando que es por medio de ella que hay que luchar contra el aborto. Sociólogos y psicólogos agregan que la educación sexual es un pre-requisito y los más radicales denuncian las desigualdades económicas como el origen del problema que debemos combatir.

Diversos grupos de opinión se pronuncian públicamente en contra del aborto y hacen saber a la mujer por diferentes medios, que el aborto no solamente es un pecado y un crimen de acuerdo con la religión católica y las leyes del país, sino que es peligroso para la salud y la vida de la mujer. A pesar de lo anterior, las mujeres siguen abortando y en ocasiones arriesgando sus vidas.

Clase de información recibida por mujeres que luego abortaron (5)

$\%$

49.2 Prohibición moral y religiosa

"El aborto es pecado"

Peligros para la salud física y mental.

El aborto es un crimen castigado por la ley

Habían recibido información en contra.

Desaprobaban el aborto.

¿Hablando en contra de él? (5)

$\begin{array}{llc} & \text { Información } & \text { Opinión } \\ & \text { Recibida } & \text { Personal } \\ & \% & \% \\ \text { Pecado } & 49.2 & 9.5 \\ \text { Peligro/Salud } & 17.6 & 49.8 \\ \text { Más pecado es tener un hijo indeseado } & 41.3\end{array}$

¿En relación con la represión legal del aborto, qué efectos ha tenido una de las más represivas legislaciones del mundo, la colombiana, sobre el aborto?

Algunos autores han argumentado que no corresponde al médico buscar las soluciones sino a las fuerzas de policía, a los educadores o la Iglesia, el hacer lo que puedan para evitar las graves consecuencias del aborto clandestino.

No hay duda que todos esos grupos se han opuesto por muchos años al aborto. ¿Cuáles son sus logros?

Los datos en la Tabla No. 2 provienen de publicaciones de la Policía Nacional de Colombia en los últimos años.

Tabla No. 2

\section{ESTADISTICAS DE CRIMINALIDAD}

\begin{tabular}{|lcccc|}
\hline & 1980 & 1983 & 1986 & 1989 \\
\hline $\begin{array}{l}\text { Abortos registrados } \\
\text { oficialmente }\end{array}$ & 136 & 119 & 110 & 86 \\
Capturas por aborto & 58 & 43 & 48 & 25 \\
\hline
\end{tabular}

Por otra parte (Tabla No. 3), y a partir de datos procedentes de la misma fuente pero teniendo en cuenta el indudable subregistro, es válido preguntarnos ¿cuántos infanticidios 0 abandono de los hijos pueden haber sido causados por un embarazo indeseado?

Tabla No. 3 ESTADISTICAS DE CRIMINALIDAD

\begin{tabular}{|lcccc|}
\hline & 1980 & 1983 & 1986 & 1989 \\
\hline Infanticidio & 65 & 22 & 15 & 15 \\
$\begin{array}{l}\text { Abandono de hijos } \\
\text { por violación }\end{array}$ & 136 & 73 & 111 & 89 \\
\hline
\end{tabular}

Regresamos así inevitablemente a la planificación familiar y a los métodos anticonceptivos como una importante medida, si bien no la única, de disminuir la morbilidad y la mortalidad ocasionada por el aborto ilegal y clandestino.

Gracias al esfuerzo de muchos profesionales y entidades la mayoría de ellas privadas, actualmente Colombia muestra orgullosamente tasas de prevalencia en el uso de anticonceptivos cercanas a las de los países desarrollados.

¿Por qué entonces no ha desaparecido el embarazo indeseado y su resultante, el aborto? Una simple mirada a las encuestas de fecundidad y prevalencia o a datos de centros médicos privados, permite encontrar los múltiples motivos que llevan a la mujer a un embarazo indeseado o inoportuno, a pesar de la existencia de excelentes programas de planificación familiar que tienen limitaciones cada vez más aparentes y tremendamente difíciles de sobrepasar.

Para que la planificación familiar sea realmente efectiva en la prevención del aborto requiere que se completen varias etapas de un proceso claramente identificado desde hace muchos años.

\section{Conocimiento de la existencia del método Uso del método escogido Uso correcto Uso Continuado}

Si el proceso se interrumpe en cualquiera de ellas, la efectividad de la planificación familiar se reduce drásticamente.

Recordemos aquí que muchas de las limitaciones de la planificación familiar son totalmente ajenas a la mujer que desea utilizar los métodos anticonceptivos y que es por culpa de dichas limitaciones y no de su "abandono" o "irresponsabilidad" que se convierte en víctima de un embarazo indeseado.

\section{Limitaciones de la planificación familiar}

Barreras culturales para su aceptación:

Mala educación sexual.

Negación de la sexualidad

Machismo.

Factores religiosos.

\section{Limitaciones técnicas:}

Inefectividad.

Intolerancia. 
Políticas gubernamentales ineficientes.

Otros obstáculos al uso adecuado de la anticoncepción, si bien directamente relacionados con la mujer, son también fruto de una cultura que estigmatiza el sexo y todo lo que con él tenga que ver directamente, como la anticoncepción y sus métodos más efectivos llamados antinaturales.

A la pregunta: “¿Por qué no habían utilizado un método anticonceptivo?"

Seiscientas dos mujeres que habían abortado, respondieron: (5)

\begin{tabular}{|c|c|c|c|}
\hline & $\%$ & & $\%$ \\
\hline * Descuido & 23.4 & Infrecuencia coital: & 22.3 \\
\hline \multicolumn{2}{|c|}{ No creyó embarazarse 19.5} & $\begin{array}{l}\text { Problemas o temores } \\
\text { ref/a salud }\end{array}$ & 11.3 \\
\hline Deseo de embarazo & 3.5 & Oposición compañero: & 8.5 \\
\hline Primera relación & 1.6 & Violación & 1.2 \\
\hline Otros & 8.7 & & \\
\hline
\end{tabular}

Sin embargo, ante la pregunta sobre conocimiento y uso de los anticonceptivos las mismas mujeres afirmaron:

\begin{tabular}{ll} 
Conocen algún método & $\%$ \\
Lo habían usado alguna vez & 100 \\
Estaban usando anticoncepción & 86.5 \\
Dicen conocer el uso correcto & 55.9 \\
Usaban correctamente el método & 35.5 \\
\hline
\end{tabular}

Es lamentable que el conocimiento de los anticonceptivos, compartido hoy por la inmensa mayoría de las mujeres colombianas de las grandes ciudades, no conduce necesariamente a su utilización y mucho menos al uso correcto de los mismos.

El origen de esta aparente inconsecuencia puede muy bien estar en relaciones de pareja donde el tabú hacia el sexo, fuertemente estimulado por nuestra cultura tradicional y por diversos grupos, impide que el tema de la anticoncepción sea siquiera discutido y por lo tanto, mucho menos llevado a la práctica.

El mismo estudio que he venido citando puso de presente la influencia que la mala relación dentro de una pareja sexual formalmente o no constituida, puede tener como obstáculo en la adopción y práctica adecuada de la planificación familiar. Al investigar sobre este punto encontramos:

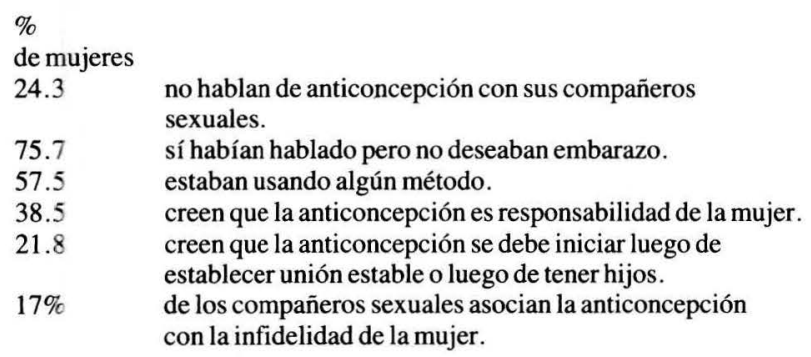

Que los métodos anticonceptivos fallan, no es misterio para nadie incluso en culturas mucho más avanzadas que la nuestra, pero cuando una mujer nos dice que está embarazada, sin quererlo la culpamos de irresponsable sin darnos cuenta que la falla anticonceptiva es una de las causas principales que da lugar más tarde al aborto con todas sus secuelas.
La efectividad en el uso, en contraste con la efectividad teórica de los métodos, queda dramáticamente registrada en el estudio de mujeres que abortaron seguramente la mayoría de ellas a consecuencia de un embarazo indeseado, Tabla No. $4,(5)$

Tabla No. 4

¿QUE METODO FALLO?

\begin{tabular}{|llll|}
\hline & $\%$ & $\%$ \\
\hline Ritmo & 21 & Diu & 9.2 \\
Ov. vagin/ & 20.4 & Retiro & 5.9 \\
Condon & 15.8 & Inyect/ & 4.9 \\
Orales & 13.8 & Quirurg/ & 1.4 \\
\hline \multicolumn{3}{|c|}{ Otros 6.9\% } \\
\hline
\end{tabular}

En Colombia, al igual que en todos los países llamados en desarrollo, vemos que el aborto es, si no la principal, una de las primeras causas de mortalidad materna. ¿Pero tiene esto necesariamente que ser así? ¿Qué sucede en países donde el aborto está ampliamente disponible para toda mujer, que actuando en forma responsable con ella misma toma la decisión de interrumpir un embarazo que no buscó ni desea?

Para ilustrar nuestro argumento sobre la inocuidad del aborto técnicamente inducido en contraste con el parto o con una de las más peligrosas complicaciones del embarazo como es su localización ectópica, veamos cuál es la mortalidad de cada uno de ellos en los Estados Unidos de Norte América. Tabla No. 5 (7)

\section{Tabla No. 5 \\ MORTALIDAD MATERNA POR CADA 100.000 CASOS USA}

\begin{tabular}{|cccc|}
\hline Edad & Emb. Ectópico & Parto & Aborto \\
\hline $15-19$ & 119 & 6.0 & 0.5 \\
$20-24$ & 56 & 6.4 & 0.3 \\
$25-29$ & 28 & 6.4 & 0.3 \\
$25-29$ & 28 & 6.4 & 0.4 \\
$30-34$ & 41 & 9.1 & 0.4 \\
$35-39$ & 53 & 19.1 & 0.4 \\
$40-44$ & 71 & 42.9 & 0.4 \\
\hline Promedio de muertes & & & \\
por cada 100.000 & 49 & 8 & 0.4 \\
casos. & & & \\
\hline
\end{tabular}

No cerremos los ojos a la realidad ni ignoremos lo que es tan evidente: Mientras el aborto técnicamente realizado acarrea CUATRO muertes maternas por cada millón de intervenciones, el aborto ilegal y clandestino como se practica en Colombia, produce entre CIEN y QUINIENTAS defunciones.

Todo lo hasta aquí escrito es apenas una parte de la problemática relacionada con el comportamiento reproductivo de la mujer colombiana, sus deseos y posibilidades de evitar un embarazo y sus fracasos en el logro de ese empeño.

¿Qué representa para nosotros como médicos, como profesionales que por escogencia estamos dedicando gran parte de nuestra vida al servicio de la mujer, a su salud, a su calidad de vida y a evitar su muerte prematura por causas relacionadas con la reproducción? 
¿Cómo podemos contribuir a la disminución de la morbilidad y la mortalidad por aborto?

* ¿Ignorando la existencia del problema?

* ¿Actuando?

$$
\text { ¿En qué forma? }
$$

¿En el consultorio?

¿En la cátedra?

¿En la política?

A pesar de los ya numerosos estudios sobre aborto publicados en la literatura médica colombiana desde hace más de 25 años, su ocasional aparición no hace justicia a la gravedad del problema en todas sus dimensiones.

Me atrevo por lo tanto a afirmar que los obstetras y ginecólogos colombianos en una gran medida han ignorado su responsabilidad frente a la solución de esta importante causa de la morbilidad y mortalidad materna, posiblemente por el hecho que la mayoría de ellos por atender un grupo poblacional de altos ingresos y suficiente educación no se ven diariamente enfrentados al embarazo indeseado y casi nunca a la muerte por aborto.

Para aquellos que tienen conciencia del problema y quieran aventurarse en la exploración de sus posibilidades de ayuda o comprometerse directamente en acciones tendientes a la solución de tan importante problema, existen múltiples alternativas desde cualquiera que sea su campo de acción profesional.

* Conociendo mejor:

No sólo el aborto ya ocurrido sino

el EMBARAZO INDESEADO QUE LO ORIGINA.

- Su magnitud.

- Sus características sociales además de médicas.

- Sus consecuencias:

No solamente médicas sino psicológicas,

familiares, sociales, demográficas.

Entendiendo que el problema no es solamente el aborto tal como se presenta en las salas de sépticas de los hospitales, sino también el embarazo indeseado.

Embarazo indeseado que es cada vez más una cruel realidad para la mujer colombiana, que por múltiples razones socio-económicas y culturales ha modificado sus ideales reproductivos y no desea en este final de siglo más de tres hijos, viviendo nueve décimas partes de su vida reproductiva ante el temor y el pánico de un embarazo que no desea.

Un simple cálculo nos permite apreciar la realidad de lo que estamos afirmando:

La vida fértil de la mujer promedio tiene una duración de 360 meses calculados entre los catorce y los 44 años. Si a esta suma restamos el número de meses empleados en los 3 hijos promedio que la mujer colombiana dice desear de acuerdo con las encuestas de fecundidad recientes y sustraemos también tres meses más de lactancia por cada hijo deseado, tenemos un total de 36 meses de su vida fértil en que podríamos afirmar que la mujer no tuvo temor del embarazo. El resto es decir 324 meses de su vida, la mujer vive bajo la continua amenaza de un embarazo que no desea.
La mujer que finalmente queda embarazada contra su voluntad, reconoce ante sí misma y algunas veces ante los demás, que su embarazo no era deseado, tal como ocurrió al $48 \%$ de las mujeres registradas en el reciente estudio de aborto hospitalario de la Federación Colombiana de Obstetricia y Ginecología. (2)

¿Quiénes son las miles de mujeres que diariamente se enfrentan a un embarazo indeseado? Ciertamente y en forma muy clara para quienes hemos indagado en este campo, no son en su mayoría jóvenes solteras supuestamente "irresponsables". Las víctimas del embarazo indeseado y candidatas al aborto con todas sus peligrosas consecuencias impuestas por la ilegalidad y la clandestinidad, son:

* Adolescentes que inician relaciones sexuales sin anticoncepción.

* Mujeres adultas que rechazan la anticoncepción por razones psicológicas o culturales.

* Mujeres de todas las edades a quienes les falla el método anticonceptivo que estaban utilizando.

* Víctimas de la violencia sexual en todos sus matices.

* Mujeres con embarazo inicialmente deseado que por razones médicas o sociales se convierte en indeseado.

El especialista en obstetricia y ginecología puede contribuir a la solución del problema creado por el embarazo indeseado y el aborto: En su consultorio, desde la cátedra, estimulando el conocimiento y el análisis integral del problema; en la política que como simples ciudadanos o desde posiciones de influencia siempre podemos y debemos ejercer promoviendo una toma de posición gubernamental consecuente con la realidad y desde luego, capacitándose adecuadamente en el manejo integral del embarazo indeseado.

Como alternativa a nuestra acción y al persistir las condiciones legales hoy día vigentes, sólo podemos esperar el agravamiento del problema y la continuación de muertes totalmente evitables.

No es exclusivo de Colombia el fracaso en la prevención del aborto inducido en condiciones inadecuadas, por medio de medidas represivas o a través de programas educativos que solamente después de muchísimos años comienzan a ser parcialmente efectivos.

Este fracaso ha sido la constante ocurrencia en países que por factores socio-demográficos similares, se vieron abocados mucho antes que nosotros a resolver ese gravísimo problema y optaron por la despenalización del aborto.

A pesar de avances recientes en la manera de pensar de nuestros legisladores, no es probable que el aborto se despenalice en Colombia en un futuro cercano y parece ser en respuesta a la inercia de los gobiernos, que hace solamente un año las dos entidades mundiales más directamente relacionadas con el médico obstetra-ginecólogo, LA ORGANIZACION MUNDIAL DE LA SALUD y la FEDERACION INTERNACIONAL DE GINECOLOGIA Y OBSTETRICIA, se pronunciaron claramente sobre nuestra responsabilidad al declarar: (6).

"El tema del aborto produce preguntas profundas y dolorosas que la mayor parte de las personas prefieren ignorar o hacerse indiferentes, pero los ginecólogos y obstetras no pueden dejar pasar el 
problema ya que sus consecuencias pueden ser funestas".

Ya hemos visto en términos generales como puede el obstetra-ginecólogo contribuir a la disminución de la mortalidad y morbilidad por aborto. Para terminar, veamos algunas acciones concretas que están a nuestra disposición inmediata:

\section{PREGUNTA USTED A todas SUS PACIENTES:}

* “Está utilizando algún método anticonceptivo?"

* (En caso negativo): “¿Desea usted quedar embarazada?"

* En caso positivo: ¿Indaga usted por el método y lo correcto de su utilización?

\section{CUANDO SU PACIENTE EMBARAZADA PRESENTA SINTOMAS DE ABORTO:}

* ¿Asume usted que ELLA desea continuar el embarazo?

* ¿Busca usted a toda costa proteger el embarazo, o consulta a su paciente sobre lo que ella prefiere?

\section{¿ESTA USTED CAPACITADO PARA AYUDAR A SU} PACIENTE?

* ¿Hace lo posible por comprenderla, apoyarla psicológicamente y ayudarla, o por el contrario la culpabiliza y la entrega involuntariamente en manos de personas irresponsables?

\section{EN RESUMEN:}

1. La mortalidad materna continúa siendo muy ELEVADA.

2. El aborto inducido es su causa principal.

3. Estas muertes son casi totalmente PREVENIBLES.

4. La planificación familiar NO ES LA SOLUCION ABSOLUTA.

5. ¿Qué puede hacer el gineco-obstetra para disminuir la morbilidad y mortalidad por aborto?

\section{¿QUE PUEDE HACER USTED?}

\section{BIBLIOGRAFIA}

1. Cataño LO y Quintero C. Estudios de Mortalidad Materna y Perinatal en Medellín y en Cali, presentados en el XVIII Congreso de OG, Barranquilla, diciembre 1991.

2. Pardo F, Uriza G. Estudio de Morbilidad y Mortalidad por Aborto en 36 instituciones de Bolivia, Colombia, Perú y Venezuela. Rev. Col. de Obstet. y Ginecol. 1991; 42 (4): 287-97

3. Encuesta de actitudes sobre el aborto y su legislación. Rev. Col Obstet. y Ginecol. 1980; 31 (1): 14-22

4. U.S. National Center for Health Statistics. 1990
5. Mora G, y col. Embarazo indeseado y aborto en Colombia ORIENTAME Bogotá, 1991. En prensa

6. Estadísticas de Criminalidad. Centro de Investigaciones Criminológicas. Policía Nacional. Fondo Rotatorio de la Policía Nacional. Serie de publicaciones $1983,1986,1989$.

7. Harlap, Susan et al. Preventing Pregnancy, Protecting Health The Alan Guttmacher Institute, New York 1990

8. FOCUS. "El aborto una crisis en progreso" OMS-FIGO, 1990. Rev Col. Obstet. y Ginecol. 1990; 41 (3):201-202 